\title{
Celebrating the arXiv
}

\section{The growing popularity of preprint servers is enriching the landscape of scientific communication.}

14 August 2011 was a special day for Paul Ginsparg, a physicist at Cornell University in the USA. The date marked the twentieth anniversary of the first e-mail submission to hep-th@xxx.lanl.gov, Ginsparg's experimental open-access bulletin board for researchers in the field of highenergy physics to communicate their latest findings ${ }^{1,2}$. At the time, Ginsparg, who was working at Los Alamos National Laboratory, probably didn't realize just how popular and important his 'preprint server' would become for communicating research findings. The system has grown dramatically in size and scope over the years to become a thriving online repository called arXiv.org. Now owned by and operated at Cornell University, the arXiv (pronounced 'archive') hosts around 720,000 e-prints spanning the disciplines of physics, maths and computer science, as well as some biology and quantitative finance. According to Ginsparg, the arXiv is growing at around 75,000 new submissions a year and delivers around one million requests for full-text downloads to about 400,000 distinct users each week ${ }^{3}$.

Many in the photonics community have warmly embraced the arXiv. A significant number of new papers related to optical physics appear every week, and it's not uncommon for findings published in Nature Photonics to have been posted first on the arXiv.

It's not hard to see why the arXiv is such a popular service. The system provides researchers with a fast and convenient way to plant a flag that shows what they did and when, avoiding the hassle and time required for peer review at a conventional journal. There's also no risk of rejection due to technical misunderstandings or subjective comments from reviewers. The arXiv is an open-access service, which means users and authors are able to see all content without any registration fees or page charges. This compelling set of benefits inspired Nature Publishing Group to launch its own preprint service called Nature Precedings in $2007^{4}$.

Kishan Dholakia, a well-known photonics researcher at St Andrews University in the UK who has been using arXiv for over ten years, explains his motivation for using it: “We can obtain a 'time stamp' for key work where we believe the field is very competitive and we wish to preserve our intellectual position."

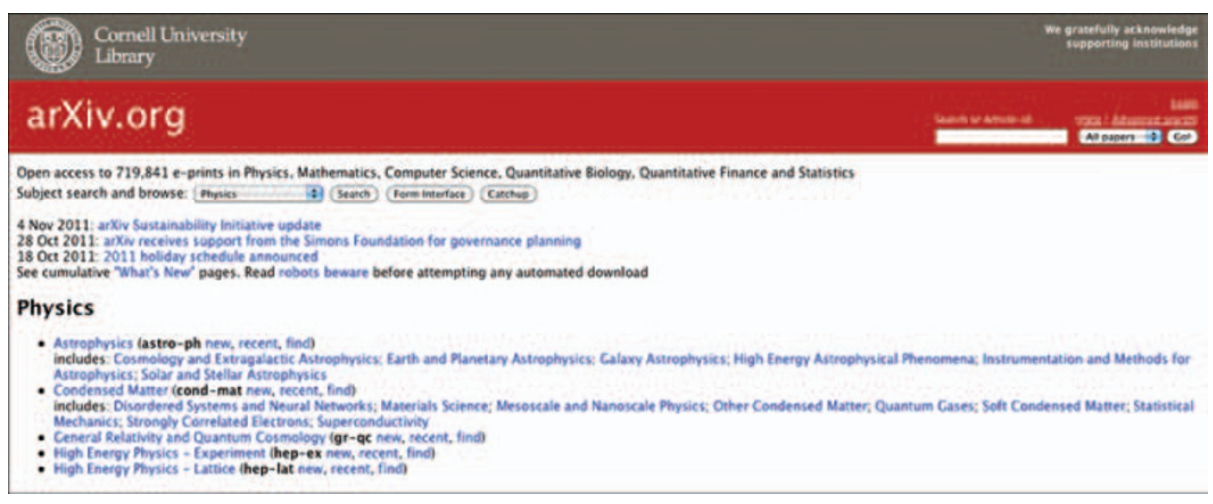

However, Dholakia doesn't post all of his results on the arXiv. "We post only our most groundbreaking results, particularly when there is intense international competition and natural uncertainty as to where and when the paper will eventually be published."

Despite its benefits, the arXiv is not a perfect system for disseminating scientific findings. The absence of a peer-review process means that uploaded material is not assessed in terms of its validity or scientific significance, which of course is an important part of publishing scientific research.

Preprint servers such as the arXiv should therefore be considered as complementary to (rather than as substitutes of) traditional academic journals. At Nature Photonics, we certainly welcome the use of preprint servers and, contrary to popular belief, our decision and opinions regarding a paper's novelty are not influenced by whether it has already appeared on a preprint server.

So are there any risks when using a preprint server such as the arXiv? Well, in principle, there's the opportunity for other researchers to be inspired by your work or even steal your ideas and submit a similar study for presentation at a conference or publication in a journal before you've had the chance to do so.

"In some sense, the arXiv may expose researchers to further risk here," comments Dholakia. "When submitting very thorough or detailed research, we are occasionally concerned that smaller studies of less scope could be published more quickly in lowerimpact journals once someone sees our work out there."

There's also the chance that a journalist may report the grand - and potentially erroneous - claims of arXiv material in a high-profile newspaper or on a website before the work has been peer-reviewed.

"The media sometimes reports stories on arXiv papers before the peer-review process, which can potentially prejudice outcomes and even skew the importance of a given work," comments Dholakia. "Furthermore, studies linked to patents cannot be submitted to arXiv, although researchers can of course submit the work for confidential peer review and withhold formal publication until a patent is filed."

It is highly likely that preprint services such as arXiv will continue to flourish and evolve, given their convenience and range of potential benefits. Ginsparg is certainly optimistic about the future opportunities of preprint publishing.

"If scholarly infrastructure can be upgraded to encourage maximal spontaneous participation, then we can expect not only increasingly automated interoperability among databases and the rising availability of materials online for algorithmic harvesting including articles, datasets, lecture notes, multimedia and software - but also qualitatively new forms of academic effort," he wrote in his recent e-print posting to celebrate arXiv's twentieth anniversary ${ }^{1}$.

"The result will be a transformation in the way we process scientific information, similar to how the availability of interlinked network resources led to new nonlinear reading strategies and the availability of networked mobile devices has altered the way we use our short- and long-term memories."

\footnotetext{
References

1. Ginsparg, P. Preprint at http://arxiv.org/abs/1108.2700v1 (2011).

2. http://physicsworld.com/cws/article/print/35983.

3. Ginsparg, P. Nature 476, 145-147 (2011).

4. http://precedings.nature.com
} 